Discussion Paper Series A No.580

\title{
Rationality and Solutions to Nonconvex Bargaining Problems: Rationalizability and Nash Solutions
}

\author{
Yongsheng Xu \\ (Department of Economics, Andrew Young School of \\ Policy Studies, Georgia State University) \\ and \\ Naoki Yoshihara \\ (Institute of Economic Research, Hitotsubashi University)
}

November, 2012

Institute of Economic Research

Hitotsubashi University

Kunitachi, Tokyo, 186-8603 Japan 


\title{
Rationality and solutions to nonconvex bargaining problems: rationalizability and Nash solutions ${ }^{1}$
}

\author{
Yongsheng Xu \\ Department of Economics \\ Andrew Young School of Policy Studies \\ Georgia State University, Atlanta, GA 30302 \\ Naoki Yoshihara \\ Institute of Economic Research \\ Hitotsubashi University, Kunitachi, Tokyo
}

This Version: August 2012

\footnotetext{
${ }^{1}$ We are grateful to Michele Lombardi for his comments and suggestions.
} 


\begin{abstract}
Conditions $\alpha$ and $\beta$ are two well-known rationality conditions in the theory of rational choice. This paper examines the implications of weaker versions of these two rationality conditions in the context of solutions to nonconvex bargaining problems. It is shown that, together with the standard axioms of efficiency and strict individual rationality, they imply rationalizability of solutions to nonconvex bargaining problems. We then characterize asymmetric Nash solutions by imposing a continuity and the scale invariance requirements. These results make a further connection between solutions to non-convex bargaining problems and rationalizability of choice function in the theory of rational choice.

J.E.L. Classification Numbers: C71, C78, D63, D71
\end{abstract}




\section{Introduction}

In this paper, we study solutions to non-convex bargaining problem by examining their connections to two well-known rationality conditions, namely conditions $\alpha$ and $\beta$, in the theory of rational choice (see, for example, Sen (1971)). Condition $\alpha$ says that, when a set $A$ contracts to another set $B$, and if an option $x$ chosen from $A$ continues to be available in $B$, then $x$ must be chosen from $B$. Condition $\beta$, on the other hand, says that, when two options $x$ and $y$ are chosen from a set $A$ and when $A$ expands to another set $B$, then, either both $x$ and $y$ are chosen from $B$ or neither $x$ nor $y$ are chosen from $B$.

In the literature on non-convex bargaining problems, a stronger version of condition $\alpha$, often called contraction independence, has been used for characterizing the Nash solution (see, for example, Kaneko (1980), Mariotti (1998, 1999), Peters and Vermeulen (2010), and Xu and Yoshihara (2006)). Contraction independence requires that, when a bargaining problem $A$ shrinks to another bargaining problem $B$ and if $B$ contains some options of the solution to $A$, then the solution to $B$ coincides with the intersection of $B$ and the solution to $A$. This version of contraction independence can also be regarded as a natural generalization of Nash's independence of irrelevant alternatives (IIA) (Nash (1950)) introduced for convex bargaining problems where a solution picks a single option from a bargaining problem. ${ }^{2}$

Building on the intuitions of conditions $\alpha$ and $\beta$, in this paper, we consider weaker versions of conditions $\alpha$ and $\beta$, to be called binary condition $\alpha$ and binary condition $\beta$, respectively. Binary condition $\alpha$ requires that, for any two options $x$ and $y$, if either $x$ or $y$ is part of the solution to a bargaining problem $A$, then the solution to the bargaining problem given by the comprehensive hull (see Section 2 for a formal definition) of $x$ and $y$ must contain the intersection of $\{x, y\}$ and the solution to $A$. Binary condition $\beta$ requires that, if two options $x$ and $y$ are the only chosen alternatives from the problem of the comprehensive hull of $x$ and $y$, then when the problem is enlarged, either both belong to the solution to the enlarged problem or neither do not belong to the solution to the enlarged problem.

We will then use these two weaker rationality conditions to first study

\footnotetext{
${ }^{2}$ There are other variations of the generalised Nash's IIA. Mariotti (1998a) introduces a weaker variant of the standard Nash IIA which is solely applicable to single-valued solutions, whereas Thomson (1981) introduces a weaker variant of the contraction independence discussed in Mariotti $(1998,1999)$ and Xu and Yoshihara (2006).
} 
rationalizability of solutions to nonconvex bargaining problems (see Section 3 for a formal definition). The interest of studying rationalizability of bargaining solutions in the literature is two-fold. In the first place, a solution can be interpreted as a fair arbitration scheme ratified by a committee (see Mariotti (1999) and Lombardi and Mariotti (2009)), and as a consequence, it represents the majority preferences of the committee. Secondly, as argued by Peters and Wakker (1991), a solution to bargaining problems may be thought to reveal the preferences of the players involved as a group, and thus the behavior of a solution may be linked to 'revealed group preference.'

In the literature on bargaining problems, the rationalizability of solutions to convex bargaining problems has been fruitfully studied (see, among others, Peters and Wakker (1991), Bossert (1994), and Sanchez (2000)), and there is little research on the rationalizability of solutions to nonconvex bargaining problems (see, however, Denicolò and Mariotti (2000), for an exception, where they consider single-valued solutions to nonconvex bargaining problems). We show that if we restrict a solution (possibly multi-valued) to be efficient and strict individually rational, then the rationalizability of a solution is equivalent to the combination of our two weaker rationality conditions. It is worth noting an interesting feature of the revealed preference relation defined in our context for establishing our result: for any two distinct alternatives $x$ and $y$ that are both strictly positive, $x$ is revealed to be preferred to $y$ if and only if $x$ is the only solution to the bargaining problem of the comprehensive hull of $x$ and $y$. Note that, under efficiency, the solution candidates of the problem of the comprehensive hull of $x$ and $y$ are just $x$ and $y$. Therefore, the revealed preference relation defined this way is based on 'pairwise comparisons' of alternatives.

After establishing the result on rationalizability of a solution, we point out that neither efficiency nor strict individual rationality is necessary for a solution to be rationalizable: there is a solution that is rationalizable but fails to satisfy either efficiency or strict individual rationality. Meanwhile, we also show that each of the axioms, efficiency, strict individual rationality, binary $\alpha$ and binary $\beta$, is indispensable for a solution to be rationalizable.

Next, we examine the consequences of imposing scale invariance on solutions to nonconvex bargaining problems. We observe that the imposition of scale invariance on a rationalizable solution to nonconvex bargaining problems in our context is not sufficient to characterize asymmetric Nash solutions. It turns out that a continuity property on part of a solution is needed. The continuity axiom we use is fairly weak as it only restricts to behaviors 
of solutions to problems given by comprehensive hulls of two alternatives. This is in sharp contrast with the results obtained for convex problems (see, for example, Roth (1977)) and for nonconvex problems where solutions are restricted to be single-valued (see, for example, Zhou (1997)).

The remainder of the paper is organized as follows. In the following section, Section 2, we present notation and definitions. Section 3 studies the rationalizability of solutions to nonconvex bargaining problems, while Section 4 is devoted to the study of asymmetric Nash solutions. We conclude in Section 5.

\section{Notation and definitions}

Let $N=\{1,2, \ldots, n\}$ be the set of all individuals in the society. Let $\mathbb{R}_{+}$be the set of all non-negative real numbers, and $\mathbb{R}_{++}$be the set of all positive numbers. Let $\mathbb{R}_{+}^{n}$ (resp. $\mathbb{R}_{++}^{n}$ ) be the $n$-fold Cartesian product of $\mathbb{R}_{+}$(resp. $\left.\mathbb{R}_{++}\right)$. For any $x, y \in \mathbb{R}_{+}^{n}$, we write $x \geq y$ to mean $\left[x_{i} \geq y_{i}\right.$ for all $\left.i \in N\right]$, $x>y$ to mean $\left[x_{i} \geq y_{i}\right.$ for all $i \in N$ and $\left.x \neq y\right]$, and $x \gg y$ to mean $\left[x_{i}>y_{i}\right.$ for all $i \in N]$. For any $x \in \mathbb{R}_{+}^{n}$ and any non-negative number $q$, we write $z=\left(q ; \mathbf{x}_{-i}\right) \in \mathbb{R}_{+}^{n}$ to mean that $z_{i}=q$ and $z_{j}=x_{j}$ for all $j \in N \backslash\{i\}$.

For any subset $A \subseteq \mathbb{R}_{+}^{n}, A$ is said to be (i) non-trivial if there exists $a \in A$ such that $a \gg 0$, and (ii) comprehensive if for all $x, y \in \mathbb{R}_{+}^{n},[x \geq y$ and $x \in A]$ implies $y \in A$. For all $A \subseteq \mathbb{R}_{+}^{n}$, define the comprehensive hull of $A$, to be denoted by $\operatorname{comp} A$, as follows:

$$
\operatorname{comp} A \equiv\left\{z \in \mathbb{R}_{+}^{n} \mid z \leq x \text { for some } x \in A\right\} .
$$

Let $\Sigma$ be the set of all non-trivial, compact and comprehensive subsets of $\mathbb{R}_{+}^{n}$. Elements in $\Sigma$ are interpreted as (normalized) bargaining problems. A bargaining solution $F$ assigns a nonempty subset $F(A)$ of $A$ for every bargaining problem $A \in \Sigma$.

\section{Rationalizable solutions}

In this section, we study the problem of rationalizability of solutions to nonconvex problems. First, we define the notion of rationalizable solutions in our context. 
Definition 1: A bargaining solution $F$ over $\Sigma$ is rationalizable if there exists a reflexive, complete and transitive binary relation $R$ over $\mathbb{R}_{+}^{n}$ such that, for all $A \in \Sigma, F(A)=\{x \in A \mid x R y$ for all $y \in A\}$.

Under what condition is a solution $F$ over $\Sigma$ rationalizable? To answer this question, we begin by introducing some axioms to be imposed on a solution to nonconvex bargaining problems. The first two, Efficiency and Strict Individual Rationality, are well-known in the literature.

Efficiency (E): For any $A \in \Sigma$ and any $a \in F(A)$, there is no $x \in A$ such that $x>a$.

Strict Individual Rationality (SIR): For all $A \in \Sigma, x \in F(A) \Rightarrow x \gg 0$.

In the literature on Nash bargaining problems and on rational choice theory, various contraction independence properties have been proposed. The idea behind a contraction independence property is the following: given two bargaining problems, $A$ and $B$, in which $A$ is a subset of $B$, and suppose that a point $x$ chosen from $B$ as a solution to $B$ continues to be available in $A$, then $x$ should continue to be a solution to $A$ provided certain restrictions are satisfied. The following axiom, to be called $(\mathrm{BC} \alpha)$, is a weaker version of the contraction independence used in nonconvex bargaining problems. It requires that, for any two points $x$ and $y$ in a bargaining problem $A$, if either $x$ or $y$ is part of the solution to $A$, then the common points in $\{x, y\}$ and the solution to $A$ must be contained in the solution to the problem given by the comprehensive hull of $x$ and $y$. It may be noted that the origin of $(\mathrm{BC} \alpha)$ goes back to Herzberger (1973) (see also Sen (1977)) where a similar condition is introduced for finite choice problems: if an option $x$ is chosen from a set $A$ then $x$ must be chosen from any two-element set $\{x, y\}$ as long as $y$ is contained in $A$ as well. Clearly, $(\mathrm{BC} \alpha)$ is also weaker than condition $\alpha$ in the literature on rational choice theory (also known as the Chernoff condition, see Chernoff (1954) and Sen (1971)). Formally, (BC $\alpha)$ is stated as follows.

Binary Condition $\alpha(\mathbf{B C} \alpha)$ : For all $A \in \Sigma$ and all $x, y \in A$, if $\{x, y\} \cap$ $F(A) \neq \varnothing$ then $F(A) \cap\{x, y\} \subseteq F(\operatorname{comp}\{x, y\})$.

It may be noted that $(\mathrm{BC} \alpha)$ is specific to non-convex bargaining problems and is not applicable to convex bargaining problems. We next introduce a weaker version of condition $\beta$ (see Sen (1971)). 
Binary Condition $\beta(\mathbf{B C} \beta)$ : For all $A \in \Sigma$ and all $x, y \in A$, if $\{x, y\}=$ $F(\operatorname{comp}\{x, y\})$, then $[x \in F(A) \Leftrightarrow y \in F(A)]$.

Thus, $(\mathrm{BC} \beta)$ requires that, whenever the solution to the problem $\operatorname{comp}\{x, y\}$ consists of both $x$ and $y$, then, for any problem $A$ containing both $x$ and $y$, either $[x$ and $y$ are both chosen as solutions to $A$ ] or [neither $x$ nor $y$ is chosen as a solution to $A]$. In a way, $(\mathrm{BC} \beta)$ stipulates that whenever two alternatives, $x$ and $y$, are "informationally equivalent" in a pairwise comparison, then they must be treated "equally".

With the help of the above axioms, we now state and prove our first main result.

Theorem 1. Let a solution $F$ over $\Sigma$ satisfy (E) and (SIR). Then, $F$ satisfies $(\mathrm{BC} \alpha)$ and $(\mathrm{BC} \beta)$ if and only if $F$ is rationalizable.

Proof. We note that if a solution $F$ over $\Sigma$ is rationalizable, then $F$ satisfies both $(\mathrm{BC} \alpha)$ and $(\mathrm{BC} \beta)$. Therefore, we need only to show that if a solution $F$ over $\Sigma$ satisfies (E), (SIR), $(\mathrm{BC} \alpha)$ and $(\mathrm{BC} \beta)$, then it must be rationalizable.

Let a solution $F$ over $\Sigma$ satisfy (E), (SIR), $(\mathrm{BC} \alpha)$ and $(\mathrm{BC} \beta)$. Define a binary relation $R$ over $\mathbb{R}_{+}^{n}$ as follows: for all $x, y \in \mathbb{R}_{+}^{n}$,

$$
\begin{aligned}
& \text { if } x=y \text {, then } x R x ; \\
& \text { if } x \neq y \text {, then } x R y \Leftrightarrow[x \in F(\operatorname{comp}\{x, y\})] \text { or }[y \notin F(A) \text { for all } \\
& A \in \Sigma \text { with } x, y \in A] .
\end{aligned}
$$

We first note that, for any $x, y \in \mathbb{R}_{+}^{n}$, if $y_{i}=0$ for some $i \in N$, then by (SIR), $y \notin F(A)$ for all $A \in \Sigma$ with $x, y \in A$. Therefore, for any $x, y \in \mathbb{R}_{+}^{n}$, $\left[y_{i}=0\right.$ for some $\left.i \in N\right] \Rightarrow x R y$. Further, if $x \gg 0$ and $y_{i}=0$ for some $i \in N$, then, by (E) and (SIR), $\{x\}=F(\operatorname{comp}\{x, y\})$ implying that $x R y$ and $\operatorname{not}(y R x)$. Therefore, the binary relation $R$ is well-defined.

Note that $R$ thus defined is reflexive and complete. We now show that $R$ is transitive. To see that $R$ is transitive, consider $x, y, z \in \mathbb{R}_{+}^{n}$ such that $x R y$ and $y R z$. If $x_{i}=0$ for some $i \in N$, then $x R y$ implies that $y_{j}=0$ for some $j \in N$, and $y R z$ together with $\left[y_{j}=0\right.$ for some $\left.j \in N\right]$ implies $z_{k}=0$ for some $k \in N$. Therefore, $x R z$ follows from the definition of $R$. If $y_{i}=0$ for some $i \in N$, then $y R z$ implies that $z_{j}=0$ for some $j \in N$. In this case, $x R z$ follows from the definition of $R$. If $z_{i}=0$ for some $i \in N$, then $x R z$ follows again from the definition of $R$. 
Consider therefore that $x \gg 0, y \gg 0$ and $z \gg 0$. Given that $x R y$ and $y R z$, it must be the case that $x \in F(\operatorname{comp}\{x, y\})$ and $y \in F(\operatorname{comp}\{y, z\})$. We need to show that $x \in F(\operatorname{comp}\{x, z\})$. Suppose to the contrary that $x \notin F(\operatorname{comp}\{x, z\})$. By $(\mathrm{E}),\{z\}=F(\operatorname{comp}\{x, z\})$. Consider the problem $\operatorname{comp}\{x, y, z\}$. Note that $\operatorname{comp}\{x, y, z\} \in \Sigma$. Consider $F(\operatorname{comp}\{x, y, z\})$. By $(\mathrm{E}), F(\operatorname{comp}\{x, y, z\}) \subseteq\{x, y, z\}$. If $x \in F(\operatorname{comp}\{x, y, z\})$, noting that $\{x, z\} \cap F(\operatorname{comp}\{x, y, z\}) \neq \varnothing$, it follows from $(\mathrm{BC} \alpha)$ that $F(\operatorname{comp}\{x, y, z\}) \cap$ $\{x, z\} \subseteq F(\operatorname{comp}\{x, z\})$, that is, $x \in F(\operatorname{comp}\{x, z\})$, a contradiction. Therefore, $x \notin F(\operatorname{comp}\{x, y, z\})$. If $y \in F(\operatorname{comp}\{x, y, z\})$, noting that $x \in$ $F(\operatorname{comp}\{x, y\})$ by $x R y$ and $(\mathrm{BC} \alpha)$, we must have $F(\operatorname{comp}\{x, y\})=\{x, y\}$ by $(\mathrm{BC} \alpha)$. It then follows from $(\mathrm{BC} \beta)$ that $x \in F(\operatorname{comp}\{x, y, z\})$, which, from the above, leads to a contradiction. Therefore, $y \notin F(\operatorname{comp}\{x, y, z\})$. If $z \in F(\operatorname{comp}\{x, y, z\})$, noting that $y \in F(\operatorname{comp}\{y, z\})$ by $y R z$ and $(\mathrm{BC} \alpha)$, it follows from $(\mathrm{BC} \alpha)$ that $F(\operatorname{comp}\{y, z\})=\{y, z\}$. It then follows from $(\mathrm{BC} \beta)$ that $y \in F(\operatorname{comp}\{x, y, z\})$, which, from the above, leads to a contradiction. Therefore, $z \notin F(\operatorname{comp}\{x, y, z\})$. Consequently, $F(\operatorname{comp}\{x, y, z\}) \cap$ $\{x, y, z\}=\varnothing$, a contradiction. Thus, it must be true that $x \in F(\operatorname{comp}\{x, z\})$ implying $x R z$. Therefore, $R$ is transitive.

To complete the proof of Theorem 1, we need to show that, for all $A \in \Sigma$, $F(A)=\{x \in A \mid x R y$ for all $y \in A\}$. Consider $A \in \Sigma$. Let $x \in F(A)$. By (SIR), for any $y \in A$ with $y_{i}=0$ for some $i \in N$, we must have $x R y$. For any $y \in A$ with $y \gg 0$, since $x \in F(A)$, by (BC $\alpha)$, it follows that $x \in F(\operatorname{comp}\{x, y\})$ implying that $x R y$. Therefore, $F(A) \subseteq\{x \in A \mid x R y$ for all $y \in A\}$. We next show that $\{x \in A \mid x R y$ for all $y \in A\} \subseteq F(A)$. Suppose, to the contrary, that it is not true that $\{x \in A \mid x R y$ for all $y \in A\} \subseteq F(A)$. Then, there must exist $x \in A$ such that $x R y$ for all $y \in A$, but $x \notin F(A)$. Note that it must be true that $x \gg 0$. Consider $z \in F(A)$. (SIR) implies that $z \gg 0$. By $(\mathrm{BC} \alpha)$ and from $z \in F(A)$ and $x \in A$, we must have $z \in F(\operatorname{comp}\{x, z\})$. Note that $x R z$, that is, $x \in F(\operatorname{comp}\{x, z\})$. Therefore, $\{x, z\}=F(\operatorname{comp}\{x, z\})$. By $(\mathrm{BC} \beta)$ and noting that $z \in F(A)$, it then follows that $x \in F(A)$, a contradiction. Therefore, $\{x \in A \mid x R y$ for all $y \in A\} \subseteq F(A)$. Hence, $F(A)=\{x \in A \mid x R y$ for all $y \in A\}$.

It may be noted that when the solution $F$ is restricted to be single-valued, we have the following result:

Corollary 1. Let a solution $F$ over $\Sigma$ be single-valued and satisfy (E) and (SIR). Then, $F$ satisfies ( $\mathrm{BC} \alpha)$ if and only if $F$ is rationalizable. 
Note that, in the above result, $(\mathrm{BC} \beta)$ is not needed since, when $F$ is single-valued, $(\mathrm{BC} \beta)$ is satisfied trivially. It may be noted that our domain is 'smaller' than the domain studied in Denicolò and Mariotti (2000) and $(\mathrm{BC} \alpha)$ is weaker than the contraction independence used by them. Therefore, Corollary 1 can be regarded as a strengthening of Theorem 1 in Denicolò and Mariotti (2000).

Remark 1. It may be noted that (E) is not necessary for a solution to be rationalizable: there exists a rationalizable solution that satisfies $(\mathrm{SIR}),(\mathrm{BC} \alpha)$ and $(\mathrm{BC} \beta)$ but violates $(\mathrm{E})$. To see this, consider the following solution: for all $A \in \Sigma$, let $F^{E}(A)=\left\{x \in A \mid x_{1}=\cdots=x_{n}\right.$, and there exists no $y \in A$ such that $y \gg x\}$ (the Egalitarian solution).

Remark 2. It is also interesting to note that (SIR) is not necessary for a solution to be rationalizable: there exists a rationalizable solution that satisfies $(\mathrm{E}),(\mathrm{BC} \alpha)$ and $(\mathrm{BC} \beta)$ but violates $(\mathrm{SIR})$. To see this, let $\geq_{\text {lex }}$ be a standard lexicographic binary relation defined over $\mathbb{R}_{+}^{n}$. Define the solution, $F^{l e x}$ as follows: for all $A \in \Sigma, F^{\text {lex }}(A)=\left\{x \in A \mid x \geq_{\text {lex }} y\right.$ for all $\left.y \in A\right\}$.

Remark 3. It may be noted that $(\mathrm{E})$ is indispensable in Theorem 1: there are non-rationalizable solutions that satisfy (SIR), $(\mathrm{BC} \alpha)$ and $(\mathrm{BC} \beta)$, but violates (E). To show this, let $\# N=2$ and for each $A \in \Sigma$, let $E(A) \equiv\left\{x \in A \mid x_{1}=x_{2}>0\right\}$. Then, define $F^{1}$ as follows: for any $A \in \Sigma$, $F^{1}(A)=F^{N}(A) \cup E(A)$, where $F^{N}=\left\{x \in A \mid \prod_{i=1}^{n} x_{i} \geq \prod_{i=1}^{n} y_{i}\right.$ for all $y \in A\}$. Note that $F^{1}$ satisfies (SIR) but violates (E). Note that, for all $\{x, y\} \in \Sigma,\{x, y\}=F^{1}(\operatorname{comp}\{x, y\})$ does not hold since $F^{1}(\operatorname{comp}\{x, y\})$ contains $E(\operatorname{comp}\{x, y\})$. Thus, $(\mathrm{BC} \beta)$ is vacuously satisfied by $F^{1} . F^{1}$ also satisfies (BC $\alpha$ ). To see this, consider $A \in \Sigma, x, y \in A$ such that $\{x, y\} \cap F^{1}(A) \neq$ $\varnothing$. Let $x, y \in F^{1}(A)$. If $x, y \in E(A)$, then $x, y \in F^{1}(\operatorname{comp}\{x, y\})$. If $x \in F^{N}(A) \backslash E(A)$ and $y \in E(A)$, then $x, y \in F^{1}(\operatorname{comp}\{x, y\})$. If $x, y \in F^{N}(A) \backslash E(A)$, then $x, y \in F^{1}(\operatorname{comp}\{x, y\})$. Let $x \in F^{1}(A)$ and $y \notin F^{1}(A)$. If $x \in E(A)$, then $x \in F^{1}(\operatorname{comp}\{x, y\})$. If $x \in F^{N}(A) \backslash E(A)$, then $x \in F^{1}(\operatorname{comp}\{x, y\})$. Therefore, $F^{1}$ satisfies $(\mathrm{BC} \alpha)$. Finally, $F^{1}$ is not rationalizable. Consider $A=\operatorname{comp}\{(4,2)\}$ and $B=\operatorname{comp}\{(4,1),(2,2)\}$. Then, $(4,2),(2,2) \in F^{1}(A)$ and $(4,1) \notin F^{1}(A)$, while $(4,1),(2,2) \in F^{1}(B)$. Suppose $F^{1}$ is rationalizable by a binary relation $R$. Then, by considering the problem $A$, we must have $(2,2) R(4,1)$ and not $(4,1) R(2,2)$, and by considering the problem $B$, we have $(2,2) R(4,1)$ and $(4,1) R(2,2)$, which is a 
contradiction. Therefore, $F^{1}$ is not rationalizable. Note that $F^{1}$ satisfies Nash's IIA and Pareto Continuity, but it is not rationalizable even within convex problems due to its multi-valuedness. This is in sharp contrast with the result obtained for convex problems. For instance, Sánchez (2000)) shows that Nash's IIA and Pareto Continuity (for this definition, see Peter and Wakker (1991) or Sánchez (2000)) are sufficient for single-valued bargaining solutions to be rationalizable in the context of convex problems.

From the above discussion, it is clear that there exists a solution that is not rationalizable (consider, for example, the solution given by the union of $F^{N}$ and $F^{E}$ ), but satisfies (SIR), $(\mathrm{BC} \alpha),(\mathrm{BC} \beta)$ and Weak Efficiency.

Remark 4. We note that (SIR) is indispensable in Theorem 1: there are nonrationalizable solutions satisfying $(\mathrm{E}),(\mathrm{BC} \alpha),(\mathrm{BC} \beta)$ and violating (SIR). To see this, let $m_{i}(A)=\max \left\{a_{i} \mid\left(a_{1}, \cdots, a_{i}, \cdots, a_{n}\right) \in A\right\}$ for all $A \in \Sigma$ and all $i \in N$. Therefore, $m(A) \equiv\left(m_{i}(A)\right)_{i \in N}$ is the ideal point of A. For each $i \in N$, let $m^{i}(A) \equiv\left(m_{i}(A) ; \mathbf{0}_{-i}\right)$. Let $P(A)$ be the set of Pareto efficient alternatives in $A \in \Sigma$. Again, let $\# N=2$. Given $A \in \Sigma$, let $\widetilde{A} \subseteq A$ be defined as follows:

$$
\widetilde{A}=\left\{x \in A \mid \forall \epsilon>0, \exists y \in \mathbb{R}_{++}^{2} \text { with }\|y-x\|<\epsilon, y \in A\right\}
$$

(For example, when $A=\operatorname{comp}\{(1,1),(2,0)\}$, then $\widetilde{A}=\operatorname{comp}\{(1,1)\}$.) Let $F^{U}(A) \equiv\left\{x \in A \mid \forall y \in A: x_{1}+x_{2} \geq y_{1}+y_{2}\right\}$ for any $A \in \Sigma$. Then, define $F^{2}$ as follows: for any $A \in \Sigma$,

1) if $\min \left\{m_{1}(A), m_{2}(A)\right\}>x_{1}+x_{2}$ for any $x \in \widetilde{A}$, then $F^{2}(A)=\left\{m^{1}(A), m^{2}(A)\right\}$;

2) if $\min \left\{m_{1}(A), m_{2}(A)\right\} \leq x_{1}+x_{2}$ for some $x \in \widetilde{A}$, but $F^{U}(A) \cap\left\{m^{1}(A), m^{2}(A)\right\} \neq$ $\varnothing$, then $F^{2}(A)=F^{U}(A) \cap\left\{m^{1}(A), m^{2}(A)\right\}$; and

3) if $F^{U}(A) \cap\left\{m^{1}(A), m^{2}(A)\right\}=\varnothing$, then $F^{2}(A)=F^{U}(A)$.

This solution satisfies (E), but violates (SIR). It also satisfies $(\mathrm{BC} \alpha)$ and $(\mathrm{BC} \beta)$. Consider $(\mathrm{BC} \alpha)$ first. Take any $A \in \Sigma$ and any $x, y \in A$, and suppose $F^{2}(A) \cap\{x, y\} \neq \varnothing$. If case 3$)$ above is applicable, then $(\mathrm{BC} \alpha)$ is obviously satisfied. If case 2) above is applicable, then $F^{2}(A) \subseteq\left\{m^{1}(A), m^{2}(A)\right\}$. Without loss of generality, let $x \in\left\{m^{1}(A), m^{2}(A)\right\}$. If $y \in\left\{m^{1}(A), m^{2}(A)\right\}$, then $\operatorname{comp}\{x, y\} \notin \Sigma$, which implies that $(\mathrm{BC} \alpha)$ is trivially satisfied. If $y \notin\left\{m^{1}(A), m^{2}(A)\right\}$ and $y \in \mathbb{R}_{+}^{2} \backslash \mathbb{R}_{++}^{2}$, again $\operatorname{comp}\{x, y\} \notin \Sigma$. If $y \notin$ $\left\{m^{1}(A), m^{2}(A)\right\}$ and $y \in \mathbb{R}_{++}^{n}$, then comp $\{x, y\} \in \Sigma$ and it corresponds to case 2$)$, so that $x \in F^{2}(\operatorname{comp}\{x, y\})$. If case 1$)$ above is applicable, then $F^{2}(A)=\left\{m^{1}(A), m^{2}(A)\right\}$. Let $x \in\left\{m^{1}(A), m^{2}(A)\right\}$ and $y \notin\left\{m^{1}(A), m^{2}(A)\right\}$. 
Then, if $y \in \widetilde{A} \cap \mathbb{R}_{++}^{2}, \operatorname{comp}\{x, y\} \in \Sigma$ and we are back to case 2). Thus, $x \in F^{2}(\operatorname{comp}\{x, y\})$. Therefore, $F^{2}$ satisfies $(\mathrm{BC} \alpha)$.

We now consider $(\mathrm{BC} \beta)$. Note that for any $A \in \Sigma$, either $F^{2}(A) \subseteq\left\{m^{1}(A), m^{2}(A)\right\}$ or $F^{2}(A) \subseteq \mathbb{R}_{++}^{2}$. Take any $A \in \Sigma$ and any $x, y \in A$, and suppose $F^{2}(\operatorname{comp}\{x, y\})=\{x, y\}$. First of all, comp $\left\{m^{1}(A), m^{2}(A)\right\} \notin \Sigma$. Moreover, if $x \in\left\{m^{1}(A), m^{2}(A)\right\}$ and $y \in \mathbb{R}_{++}^{2}$, then $F^{2}(\operatorname{comp}\{x, y\})=\{x, y\}$ does not hold. Thus, that $F^{2}(\operatorname{comp}\{x, y\})=\{x, y\}$ implies case 3$)$ is applicable. Consequently, $F^{U}(\operatorname{comp}\{x, y\})=\{x, y\}$. Therefore, $F^{2}$ satisfies $(\mathrm{BC} \beta)$.

To see that $F^{2}$ is not rationalizable, let $\triangle$ be the unit simplex, and consider $x=(0,2), y=(2,0), A=\operatorname{comp}(\{x,(3,0)\} \cup \triangle)$, and $B=\operatorname{comp}(\{y,(0,3)\} \cup \triangle)$.

Note that $x \in F^{2}(A), y \notin F^{2}(A), y \in F^{2}(B)$ and $x \notin F^{2}(B)$ implying no binary relation $R$ can be defined over $\mathbb{R}_{+}^{2}$ that will rationalize $F^{2}$. The above argument still works even if $F^{2}$ is refined to be single-valued and this refined solution satisfies all the axioms except (SIR). This observation suggests that (SIR) is indispensable for rationalizable bargaining solutions to nonconvex problems that are single-valued.

Remark 5. $(\mathrm{BC} \alpha)$ is indispensable in Theorem 1: there are non-rationalizable solutions satisfying $(\mathrm{E}),(\mathrm{SIR})$ and $(\mathrm{BC} \beta)$ but violating $(\mathrm{BC} \alpha)$. To see this, consider the lexicographic Kalai-Smorodinsky solution $F^{\text {lexKS }}$ which is defined as usual. This solution satisfies all the axioms in Theorem 1 except $(\mathrm{BC} \alpha)$ and is not rationalizable.

Remark 6. $(\mathrm{BC} \beta)$ is indispensable in Theorem 1: there are non-rationalizable solutions satisfying $(\mathrm{E}),(\mathrm{SIR})$ and $(\mathrm{BC} \alpha)$ but violating $(\mathrm{BC} \beta)$. To see this, for any $A \in \Sigma$, let $F^{P S I R}(A)=\left\{x \in A \cap \mathbb{R}_{++}^{n} \mid\right.$ there exists no $y \in A$ such that $y>x\}$. This solution satisfies all the axioms in Theorem 1 except $(\mathrm{BC} \beta)$ and is not rationalizable.

\section{Asymmetric Nash solutions}

In this section, we show that under $(\mathrm{E}),(\mathrm{SIR}),(\mathrm{BC} \alpha)$ and $(\mathrm{BC} \beta)$, if we impose Nash's scale invariance axiom and require a solution to be continuous (see the formal definition below), then we obtain an asymmetric Nash solution defined below. 
Definition 2. A bargaining solution $F$ over $\Sigma$ is an asymmetric Nash solution if there exist $t_{1}>0, \cdots$, and $t_{n}>0$ such that, for all $A \in \Sigma$, $F(A)=\left\{x \in A \mid \prod_{i=1}^{n} x_{i}^{t_{i}} \geq \prod_{i=1}^{n} y_{i}^{t_{i}}\right.$ for all $\left.y \in A\right\}$

To study asymmetric Nash solutions, we first introduce the axioms of scale invariance and continuity.

Scale Invariance (SI): For all $A \in \Sigma$ and all $t \in \mathbb{R}_{++}^{n}$, if $t A=\left\{\left(t_{i} a_{i}\right)_{i \in N}\right.$ $a \in A\}$ then $F(t A)=\left\{\left(t_{i} a_{i}\right)_{i \in N} \mid a \in F(A)\right\}$.

Continuity (CON): For any $x, y \in \mathbb{R}_{+}^{n}$ with $x \neq y$, if $\{x\}=F(\operatorname{comp}\{x, y\})$ then there exists $\epsilon>0$ such that for all $z \gg 0$ and all $z^{\prime} \in \mathbb{R}_{+}^{n}$,

$[\|z-x\|<\epsilon \Rightarrow\{z\}=F(\operatorname{comp}\{y, z\})]$ and $\left[\left\|z^{\prime}-y\right\|<\epsilon \Rightarrow\{x\}=F\left(\operatorname{comp}\left\{x, z^{\prime}\right\}\right)\right]$.

It may be noted that $(\mathrm{CON})$ introduced above is somewhat weaker than a continuity property introduced in Peters and Vermeulen (2010), but very different from various other continuity properties discussed in the literature on bargaining problems (see, for example, Kaneko (1980), Bossert (1994), Peters and Wakker (1991), and Sánchez (2000)). To a certain degree, (CON) is a fairly weak requirement as it restricts its applicability to a class of problems each consisting of the comprehensive hull of two points.

With the help of $(\mathrm{CON})$, we present and prove the second main result of our paper.

Theorem 2. A solution $F$ over $\Sigma$ satisfies (E), (SIR), (BC $\alpha),(\mathrm{BC} \beta),(\mathrm{SI})$ and (CON) if and only if it is an asymmetric Nash.

Proof. It can be checked that an asymmetric Nash solution satisfies (E), (SIR), $(\mathrm{BC} \alpha),(\mathrm{BC} \beta),(\mathrm{SI})$ and $(\mathrm{CON})$. We now show that if a solution satisfies $(\mathrm{E}),(\mathrm{SIR}),(\mathrm{BC} \alpha),(\mathrm{BC} \beta),(\mathrm{SI})$ and $(\mathrm{CON})$, then it must be an asymmetric Nash solution.

Let $F$ satisfy $(\mathrm{E}),(\mathrm{SIR}),(\mathrm{BC} \alpha),(\mathrm{BC} \beta),(\mathrm{SI})$ and $(\mathrm{CON})$. From Theorem $1, F$ is rationalizable by a reflexive, transitive and complete binary relation $R$ over $\mathbb{R}_{+}^{n}$. Define the binary relation $R$ as in the proof of Theorem 1 . Note that $F$ satisfies (CON). Then, $R$ must be continuous over $\mathbb{R}_{++}^{n}$. Since $F$ satisfies (SI), $R$ satisfies the following property: for all $x, y \in \mathbb{R}_{++}^{n}$ and 
all $\lambda \in \mathbb{R}_{++}^{n}, x R y \Leftrightarrow\left(\lambda_{1} x_{1}, \cdots, \lambda_{n} x_{n}\right) R\left(\lambda_{1} y_{1}, \cdots, \lambda_{n} y_{n}\right)$. Then, following Tsui and Weymark (1997) (see also Xu (2002)), there exist $t_{1}, \cdots, t_{n}$ such that, for all $x, y \in \mathbb{R}_{++}^{n}, x R y \Leftrightarrow \prod_{i=1}^{n} x_{i}^{t_{i}} \geq \prod_{i=1}^{n} y_{i}^{t_{i}}$. By (E), it follows that $t_{1}>0, \cdots, t_{n}>0$. Note that if $y_{i}=0$ for some $i \in N$, then $x R y$. Therefore, $R$ can be represented by a Cobb-Douglas function. Hence, $F$ is an asymmetric Nash solution. $\diamond$

It may be noted that any single-valued asymmetric Nash solution violates $(\mathrm{CON})$. Let a single-valued asymmetric Nash solution $F^{a n}$ be given as follows: for all $A \in \Sigma, F^{a n}(A) \in\left\{x \in A \mid \prod_{i=1}^{n} x_{i}^{t_{i}} \geq \prod_{i=1}^{n} y_{i}^{t_{i}}\right.$ for all $\left.y \in A\right\}$, where $t_{i}>0$ for all $i \in N$. Consider $x, y \in \mathbb{R}_{++}^{n}$ with $\prod_{i=1}^{n} x_{i}^{t_{i}}=\prod_{i=1}^{n} y_{i}^{t_{i}}$ and the problem $B=\operatorname{comp}\{x, y\}$. Without loss of generality, let $F^{a n}(B)=$ $x$. Then, for all $\epsilon>0$, there exists $z \gg 0$ with $\|z-x\|<\epsilon$, such that $F^{a n}(\operatorname{comp}\{z, y\})=y$. This implies a violation of $(\mathrm{CON})$.

In view of the above observation and from Theorem 2, we have the following impossibility result, a similar but different version is obtained in Peters and Vermeulen (2010) where they show the incompatibility of singlevaluedness of a solution with the axioms of Weak Efficiency, (SIR), (SI), Contraction Independence and their continuity property.

Proposition 1. Suppose $F$ is single-valued. Then, there is no $F$ over $\Sigma$ satisfying $(\mathrm{E}),(\mathrm{SIR}),(\mathrm{BC} \alpha),(\mathrm{BC} \beta),(\mathrm{SI})$ and $(\mathrm{CON})$.

Remark 7. Note that (CON) is indispensable in Theorem 2. Indeed, there exists a solution satisfying $(\mathrm{E}),(\mathrm{SIR}),(\mathrm{BC} \alpha),(\mathrm{BC} \beta)$ and $(\mathrm{SI})$ while violating (CON). For instance, consider the following solution: for all $A \in \Sigma$, $F^{3}(A)=\left\{x \in F^{N}(A) \mid x \geq_{\text {lex }} y\right.$ for all $\left.y \in F^{N}(A)\right\}$, where $\geq_{\text {lex }}$ is a usual lexicographic relation. This solution satisfies $(\mathrm{E}),(\mathrm{SIR}),(\mathrm{BC} \alpha),(\mathrm{BC} \beta)$, and (SI), but violates $(\mathrm{CON})$. By the definition, $F^{3}$ is neither an asymmetric Nash nor the (symmetric) Nash solution.

Remark 8. Roth (1977) uses (SIR) to derive an asymmetric Nash solution for convex bargaining problems. Zhou (1997) uses (SIR) together with Nash's IIA (which is stronger than our $(\mathrm{BC} \alpha)$ ) and $(\mathrm{SI})$ to derive an asymmetric Nash solution for nonconvex bargaining problems. In Zhou's approach, a solution is assumed to be single-valued. In our context with a multi-valued solution, if we drop (E), the solution $F^{W P-}$ which is defined as, for any $A \in \Sigma, F^{W P-}(A)=\left\{x \in A \cap \mathbb{R}_{++}^{n} \mid\right.$ there exists no $y \in A$ such that $\left.y \gg x\right\}$, satisfies (SIR), $(\mathrm{BC} \alpha),(\mathrm{BC} \beta)$, and $(\mathrm{SI})$. Thus, there are solutions other than 
asymmetric Nash solutions satisfying (SIR), $(\mathrm{BC} \alpha),(\mathrm{BC} \beta)$, and (SI). Note that $F^{W P-}$ satisfies $(\mathrm{CON})$.

Remark 9. Note that $F^{W P-}$ constructed in Remark 8 satisfies Weak Efficiency, $(\mathrm{SIR}),(\mathrm{BC} \alpha),(\mathrm{BC} \beta)$, and $(\mathrm{CON})$, and is different from any asymmetric Nash solution. Therefore, in our Theorem 2, (E) cannot be weakened to Weak Efficiency. This is in contrast with Theorem 5.2 in Peters and Vermeulen (2010) where they derive an asymmetric Nash solution by using Weak Efficiency, (SI), (SIR), Contraction Independence (see, for example, Xu and Yoshihara (2006) and Peters and Vermuelen (2010)), and their continuity property for the domain that also contains finite bargaining problems. The contrast between our Theorem 2 and Theorem 5.2 in Peters and Vermeulen (2010) suggests a trade-off between 'degrees' of rationality and 'degrees' of efficiency requirement.

Remark 10. It is interesting to note that, there are single-valued solutions satisfying $(\mathrm{E}),(\mathrm{BC} \alpha),(\mathrm{BC} \beta)$ and $(\mathrm{SI})$, which are different from asymmetric Nash solutions. Let $\geq_{\text {lex }}$ be a standard lexicographic binary relation defined over $\mathbb{R}_{+}^{n}$. Define the solution, $F^{l e x}$, as follows: for all $A \in \Sigma$, $F^{\text {lex }}(A)=\left\{x \in A \mid x \geq_{\text {lex }} y\right.$ for all $\left.y \in A\right\}$. This solution satisfies (E), $(\mathrm{BC} \alpha)$ (because the solution is rationalizable), $(\mathrm{BC} \beta)$ (because the solution is again rationalizable) and (SI). $F^{\text {lex }}$ is single-valued. Note that $F^{l e x}$ violates $(\mathrm{SIR})$ and $(\mathrm{CON})$. This suggests that $(\mathrm{E}),(\mathrm{BC} \alpha),(\mathrm{BC} \beta),(\mathrm{SI})$ and the single-valuedness of a solution are not sufficient to derive asymmetric Nash solutions.

Remark 11. It may be noted that the solution $F^{\text {lex }}$ defined in Remark 9 is rationalizable, and therefore, $F^{l e x}$ satisfies Nash's IIA (Independence of Irrelevant Alternatives). Note that $F^{l e x}$ is single-valued, and satisfies (E) and (SI), and is different from any single-valued asymmetric Nash solution. To see the difference between $F^{l e x}$ and any single-valued asymmetric Nash solution, consider the problem $A=\left\{x \in \mathbb{R}_{+}^{n} \mid x_{1}+\cdots+x_{n} \leq 1\right\}$. Then, $F^{\text {lex }} \in\{(1,0, \cdots, 0),(0,1,0, \cdots, 0), \cdots,(0, \cdots, 0,1)\}$, while any single-valued asymmetric Nash solution will choose an $x \in A$ such that $x_{i}>0$ for all $i \in N$. Therefore, $F^{l e x}$ serves as a counter-example to Theorem 2 of Denicolò and Mariotti (2000) where they stated that any single-valued asymmetric Nash solution can be derived from (E), (SI) and Nash's IIA. In view of the solution $F^{l e x}$, it is clear that (E), (SI) and Nash's IIA together do not imply any single-valued asymmetric Nash solution. 
Remark 12. In Theorem 2, if we impose Anonymity (see, for example, Xu and Yoshihara (2006), for a definition) on a solution $F$, then we obtain a characterization of the (symmetric) Nash solution. It can be verified, however, that both (SIR) and (CON) are redundant if Anonymity is added and that (E) can be replaced by Weak Efficiency. As a consequence, the symmetric Nash solution is characterized by Weak Efficiency, $(\mathrm{SI}),(\mathrm{BC} \alpha),(\mathrm{BC} \beta)$ and Anonymity.

\section{Conclusion}

In this paper, we have examined the implications of two weaker versions of conditions $\alpha$ and $\beta$ in the context of solutions to non-convex bargaining problems. In particular, we have shown that, (i) under efficiency and strict individual rationality, they are equivalent to rationalizable solutions, and (ii) together with efficiency, strict individual rationality, scale invariance and a weak continuity requirement, they characterize asymmetric Nash solutions. Conditions $\alpha$ and $\beta$, together, characterize rationalizability of a choice function defined over the set of all non-empty subsets of a finite universal set in terms of an ordering. It is therefore interesting to note that, in non-convex bargaining problems, $(\mathrm{BC} \alpha)$ and $(\mathrm{BC} \beta)$ are associated with "rationalizability" of a solution to bargaining problems. Our results clarify several issues relating to rationalizable solutions to nonconvex bargaining problems and to asymmetric Nash bargaining solutions, make further connections between two widely used rationality conditions in rational choice theory and solutions to non-convex bargaining problems, and improve characterizations of Nash solutions to nonconvex bargaining problems.

\section{References}

Bossert, W. (1994), Rational choice and two-person bargaining solutions, Journal of Mathematical Economics 23: 549-563.

Chernoff, H. (1954), Rational selection of decision functions, Econometrica 22: $422-443$.

Denicolò, V. and M. Mariotti (2000), Nash bargaining theory, nonconvex problems and social welfare orderings, Theory and Decision 48(4), 351- 
358.

Herzberger, H.G. (1973), Ordinal preference and rational choice, Econometrica 41, 187-237.

Kaneko, M. (1980), An extension of the Nash bargaining problem and the Nash social welfare function, Theory and Decision 12, 135-148.

Mariotti, M. (1998), Nash bargaining theory when the number of alternatives can be finite, Social Choice and Welfare 15, 413-421.

Mariotti, M. (1998a), Extending Nash's axioms to nonconvex problems, Games and Economic Behavior 22, 377-383.

Mariotti, M. (1999), Fair bargains: distributive justice and Nash bargaining theory, Review of Economic Studies 66, 733-741.

Nash, J. (1950), The bargaining problem, Econometrica 18: 155-162.

Peters, H. and D. Vermeulen (2010), WPO, COV and IIA bargaining solutions for non-convex bargaining problems, International Journal of Game Theory DOI 10.1007/s00182-010-0246-6.

Peters, H. and P. Wakker (1991), Independence of irrelevant alternatives and revealed groups preferences, Econometrica 59: 1787-1801.

Roth, A. (1977), Individual rationality and Nash's solution to the bargaining problem, Mathematics of Operations Research 2, 64-65.

Sánchez, M. C. (2000), Rationality of bargaining solutions, Journal of Mathematical Economics 33: 389-399.

Sen, A.K. (1971), Choice functions and revealed preference, Review of Economic Studies 38, 307-317.

Sen, A.K. (1977), Social choice theory: a re-examination, Econometrica 45, $53-88$.

Tsui, K. and J. Weymark (1997), Social welfare orderings for ratio-scale measurable utilities, Economic Theory 10, 241-256.

Thomson, W. (1981), A class of solutions to bargaining problems, Journal of Economic Theory 25, 431-441. 
$\mathrm{Xu}, \mathrm{Y}$. (2002), Functioning, capability and the standard of living an axiomatic approach, Economic Theory 20, 387-399.

$\mathrm{Xu}, \mathrm{Y}$. and N. Yoshihara (2006), Alternative characterizations of three bargaining solutions for nonconvex problems, Games and Economic Behavior 57, 86-92.

Zhou, L. (1997), The Nash bargaining theory with non-convex problems, Econometrica 65, 681-685. 